\title{
Controlled Cold Water and Water Slushy Ingestion, and Heat Performance in Subjects of Average Fitness
}

Cantu SN, Hearon CM, Bliss MV, and Fiddler RE

Human Performance Laboratory; Department of Health and Kinesiology; Texas A\&M University-Kingsville; Kingsville, TX

Category: Undergraduate
Advisor/Mentor: Hearon, C.M. (christopher.hearon@tamuk.edu)

ABSTRACT

Fluid ingestion is known to improve exercise performance and could lead to a heat sink effect, if cold enough. While research has been conducted on the influence of hydration in exercise performance, little has been done which consider beverages' temperature during controlled consumption. PURPOSE: To examine the effect of controlled consumption of water at different temperatures on heat performance in subjects of average fitness. METHODS: Fifteen males, ages 18-29, with no prior heat illness were recruited. Subjects were tested for body composition and peak oxygen consumption $\left(\mathrm{VO}_{2 \text { peak }}\right)$ prior to testing. All subjects underwent three experimental trials [cold water $(C D=4 \square C)$, water slushy ( $S L=-1 \square C)$, room temperature water $(\mathrm{RM}=22 \square \mathrm{C})]$ in a balanced crossover design. Subjects were required to exercise on a cycle ergometer at intensity $70 \% \mathrm{VO}_{2 \text { peak }}$ (vigorous exercise) in the heat $(34.0 \pm 0.6 \square \mathrm{C}, 41.7 \pm 2.7 \% \mathrm{RH}$, $3.6 \mathrm{~km} \cdot \mathrm{hr} \cdot{ }^{-1}$ wind speed) until volitional maximum. Subjects were required to consume a controlled volume ( $\left.2.5 \mathrm{~g} \cdot \mathrm{kg}_{\text {BodyMass }}{ }^{-1}\right)$ of one of the treatments (CD, SL, RM) every 10 minutes each trial.

Measurements for maximum exercise time (ExT), pre-/post-core body temperature change $\left(\Delta \mathrm{T}_{\mathrm{c}}\right)$, heart rate (HR), mean skin temperature $\left(\mathrm{MT}_{\mathrm{sk}}\right.$ ), sweat rate (SR), and RPE were recorded. One-way (beverage) or two-way (beverage $x$ time) ANOVA with repeated measures was used ( $a=0.05)$. RESULTS: ExT did not differ significantly between treatments $(C D=33.8 \pm 9.4 \mathrm{~min} ; \mathrm{SL}=35.0 \pm 9.8 \mathrm{~min} ; \mathrm{RM}=31.5 \pm 8.6 \mathrm{~min})$ but a trend $(p=0.0680)$ was seen where SL\&CD>RM, which was supported by all subjects having their longest bouts during $\mathrm{CD}(n=10)$ and SL $(n=5)$ trials. Neither $\Delta \mathrm{T}_{\mathrm{c}}\left(\mathrm{CD}=0.69 \pm 0.36^{\circ} \mathrm{C}, \mathrm{SL}=0.64 \pm 0.43^{\circ} \mathrm{C}\right.$, $\left.\mathrm{RM}=0.77 \pm 0.45^{\circ} \mathrm{C}\right)$, or SR $\left(\mathrm{CD}=1545 \pm 1109 \mathrm{ml} \cdot \mathrm{hr}^{-1} ; \mathrm{SL}=1837 \pm 692 \mathrm{ml} \cdot \mathrm{hr}^{-1} ; \mathrm{RM}=1891 \pm 489 \mathrm{ml} \cdot \mathrm{hr}^{-1}\right)$, differed $(p>0.05)$ between treatments. A main effect for beverage was seen in $\mathrm{HR}(\mathrm{CD}=157 \pm 16 \mathrm{bpm} ; \mathrm{SL}=153 \pm 18$ bpm; $\mathrm{RM}=160 \pm 17 \mathrm{bpm})(p<0.05)$ where $\mathrm{SL}<\mathrm{RM}$, but there was no significant differences in $\mathrm{MT}_{\text {sk }}$ or $\mathrm{RPE}$ $(p>0.05)$. A main effect for time $(p<0.05)$ was see in $\mathrm{HR}(\mathrm{T} 20=161 \pm 18 \mathrm{bpm}>\mathrm{T} 10=153 \pm 16 \mathrm{bpm}), \mathrm{MT}_{\mathrm{sk}}$ $\left(\mathrm{T} 20=36.2 \pm 0.3^{\circ} \mathrm{C}>\mathrm{T} 10=35.9 \pm 0.3^{\circ} \mathrm{C}\right)$, and $\operatorname{RPE}(\mathrm{T} 20=5.8 \pm 2.1(0-10)>\mathrm{T} 10=3.3 \pm 1.4(0-10))$. A trend towards significant beverage $x$ time interaction was seen for HR $(p=0.0900)$ but treatments did not respond differently over time for $\mathrm{MT}_{\text {sk }}$ or RPE $(p>0.05)$. HR at volitional maximum differed between treatments $(\mathrm{CD}=168 \pm 20 \mathrm{bpm} ; \mathrm{SL}=165 \pm 20 \mathrm{bpm} ; \mathrm{RM}=173 \pm 20 \mathrm{bpm})(p<0.05)$, specifically $\mathrm{SL}<\mathrm{RM}$, but no differences were seen between $\mathrm{MT}_{\text {sk }}$ or RPE $(p>0.05)$. CONCLUSION: SL appeared to improve performance over $\mathrm{RM}$, but not $\mathrm{CD}$. There may be a point where colder beverage temperature does not yield a greater heat sink effect or, results could have been due to shorter exercise time in subjects of average fitness. 